\title{
Dynamo-generated magnetic fields in fast rotating single giants
}

\section{Renada Konstantinova-Antova ${ }^{1}$, Michel Aurière ${ }^{2}$, Klaus-Peter Schröder ${ }^{3}$ and Pascal Petit ${ }^{2}$}

\author{
${ }^{1}$ Inst. of Astronomy, Bulgarian Acdy of Sciences, Sofia, 72 Tsarigradsko shosse, Bulgaria \\ email: renada@astro.bas.bg \\ ${ }^{2}$ Laboratoire d'Astrophysique de Toulouse-Tarbes, Université de Toulouse, CNRS, \\ Observatoire Midi Pyrénés, 57 Avenue d’Azereix, 65008 Tarbes, France \\ email: michel.auriere@ast.obs-mip.fr \\ ${ }^{3}$ Departmento de Astronomia, Universidad de Guanajuato, GTO, \\ Academic Street, Camford, CF3 5QL, Mexico \\ email: kps@astro.ugto.mx
}

\begin{abstract}
Red giants offer a good opportunity to study the interplay of magnetic fields and stellar evolution. Using the spectro-polarimeter NARVAL of the Telescope Bernard Lyot (TBL), Pic du Midi, France and the LSD technique we began a survey of magnetic fields in single G$\mathrm{K}-\mathrm{M}$ giants. Early results include $6 \mathrm{MF}$-detections with fast rotating giants, and for the first time a magnetic field was detected directly in an evolved M-giant: EK Boo. Our results could be explained in the terms of $\alpha-\omega$ dynamo operating in these giants.
\end{abstract}

Keywords. Magnetic fields - stars: evolution - stars: late-type - stars: activity

\section{Introduction}

Magnetic fields (MF) in single evolved stars are still poorly studied. Most of the $\mathrm{G}-\mathrm{K}-$ M giants presently been known as active are fast rotators. (Fekel\&Balachandran, 1993; Huensch et al., 2004). Angular momentum dredge-up has been suggested to provide the fast rotation, driven by the convective zone reaching near the stellar core (Simon\&Drake, 1989). In this way, MFs could be generated by a new dynamo.

\section{Observations and data processing}

The new generation spectro-polarimeters like NARVAL at TBL, Pic du Midi, France (Aurière, 2003) and the LSD technique (Donati et al., 1997) are very suitable for precision detections of MFs in giants (Konstantinova-Antova et al., 2008; Aurière et al., 2008). We observed 7 fast rotating giants with NARVAL in the period of November 2006 to April 2008. A precise computation of the longitudinal MF $B_{l}$ using the First Moment Method (Donati et al., 1997; Rees \& Semel, 1979) was carried out, and the time-behavior of the activity indicator CaII K (S index) was studied.

\section{First results}

MF structures, indicative of a dynamo, were detected for 6 of the 7 giants (exception: HD233517), see Table 1 listing the $\mathrm{S}$ index and $B_{l}$. We determined the evolutionary status and masses of these 6 giants, using Hipparcos parallaxes, $T_{\text {eff }}$ from the Wright catalogue (2003), and matching evolutionary tracks from Schröder et al. (1997) (Fig. 1).

All 6 giants with MF-detection have masses $\geqslant 1.5 M_{\odot}$ and their convective zones are currently deepening while the stars are evolving. Their evolutionary stages reach from the 
Table 1. Data for the studied giants.

\begin{tabular}{|c|c|c|c|c|c|c|c|}
\hline Star & Sp class & $\mathrm{M} / M_{\odot}$ & $\begin{array}{r}\text { Vsini } \\
\mathrm{km} / \mathrm{s}\end{array}$ & $\begin{array}{c}L_{x} \\
10^{30} \mathrm{erg} / \mathrm{s}\end{array}$ & $\begin{array}{c}\text { CaII K } \\
\text { S index }\end{array}$ & $\begin{array}{c}B_{l} \\
\text { Gauss }\end{array}$ & $\begin{array}{c}\text { error } B_{l} \\
\text { Gauss }\end{array}$ \\
\hline V390 Aur & G8III & 1.85 & 29 & 5.04 & 0.64 & $-5--15$ & 4.4 \\
\hline FI Cnc & G8III & 2.25 & 17 & 26 & $1.04-1.35$ & $-16.48-+3.45$ & 1.87 \\
\hline 37 Com & G9III-II & 4.2 & 11 & 5.20 & 0.34 & +5.62 & 0.63 \\
\hline 7 Boo & G5III & 3.6 & 14.5 & 3.72 & 0.24 & +1.83 & 0.84 \\
\hline$\kappa$ HerA & G8III & 2.8 & 9.4 & 2.98 & 0.29 & -3.94 & 0.67 \\
\hline HD233517 & K2III & $1.5 ?$ & 15 & & & no detect. & \\
\hline EK Boo & M6III & 1.9 & 11 & 14.12 & $0.21-0.26$ & $-3.19--6.76$ & 0.62 \\
\hline
\end{tabular}

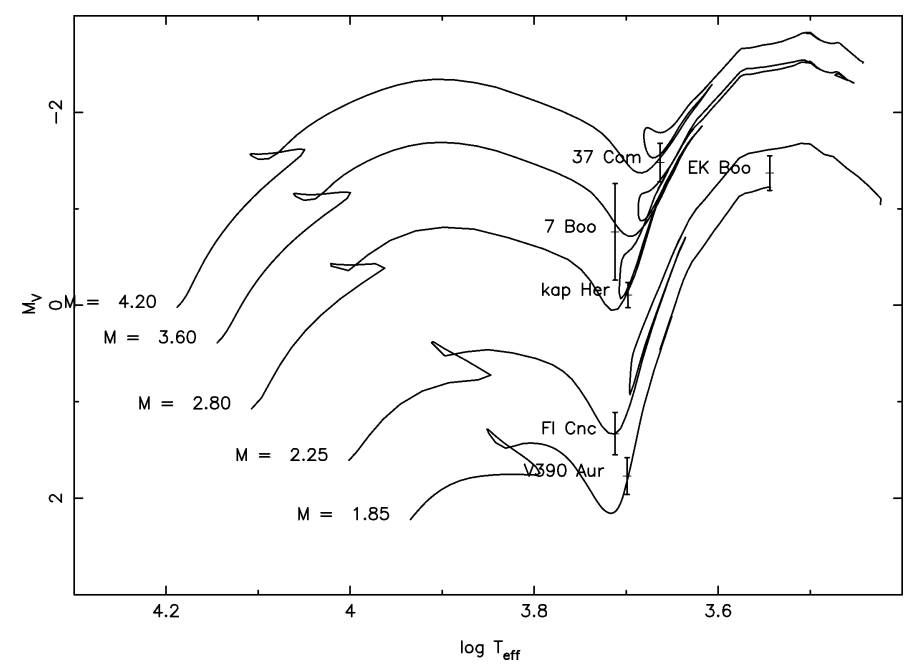

Figure 1. Situation of the fast rotating giants on the HR diagram.

Hertzsprung gap to the AGB. While the particular reasons for the fast rotation could be different, depending on mass and evolutionary history, an $\alpha-\omega$ dynamo presents a likely reason for the detected magnetic activity.

\section{Acknowledgements}

We thank the TBL team for providing the observations, a substantial part was supported by the OPTICON programme. The Hipparcos database was used.

\section{References}

Aurière M. 2003, EAS Publ. Series 9, 105

Aurière, M., Konstantinova-Antova, R. Petit, P., et al. 2008, A\&A 491, 499

Donati, J.-F., Semel, M., Carter, B. D. et al. 1997, MNRAS 291, 658

Fekel F. C. \& Balachandran S. 1993, ApJ 403, 708

Hünsch M., Konstantinova-Antova R., Schmitt J. H. M. et al. 2004, in Proc. IAU Symp. 219, A. Dupree and A. Benz (eds.), p.223

Konstantinova-Antova R., Aurière, M., Iliev I. et al. 2008, A $\mathscr{E A ~ 4 8 0 , ~} 475$

Rees D. E. \& Semel M. 1979, A\&A 74, 1

Schröder K.-P., Pols O. P., \& Eggleton P. P. 1997, MNRAS 285, 696

Simon T. \& Drake S. A. 1989, ApJ 346, 303

Wright C. O., Egan M. P., Kraemer, K. E. et al. 2003, AJ 125, 359 